\title{
Cultura de emprendimiento mediante la investigación como estrategia pedagógica apoyada en TIC
}

\section{Culture of entrepreneurship through research as a pedagogical strategy supported by TIC}

\author{
DOI: http://dx.doi.org/10.17981/cultedusoc.9.3.2018.90
}

Artículo de investigación. Fecha de recepción: 15/06/2018. Fecha de aceptación: 27/11/2018

\author{
Alexandra Bonilla-Llanes ${ }^{1}$; \\ Dioselina Botello-Trillor; Elmira Eguis-Pabó; Francia Lara-De La Rosa; \\ Nelsy Thomas-De Manjarrez; Olga Pedroza-Miranda; Rosa Ponzon-Rodríguez² \\ IED John F. Kennedy de Aracataca, sede principal (Colombia) \\ alexbonillanes@hotmail.com
}

Para citar este artículo:

Bonilla-Llanes, A., Botello-Trillor, D., Eguis-Pabó, E., Lara-De La Rosa, F., Thomas-De Manjarrez, N., Pedroza-Miranda, O. y PonzonRodríguez, R. (2018). Cultura de emprendimiento mediante la investigación como estrategia pedagógica apoyada en TIC. Cultura. Educación y Sociedad 9(3), 763-774. DOI: http://dx.doi.org/10.17981/cultedusoc.9.3.2018.90

\section{Resumen}

La cultura de emprendimiento permite fomentar pensamientos y acciones, orientadas a generar ingresos económicos, aprovechando recursos y oportunidades, basados en el desarrollo de una visión global, donde se debe gestionar un riesgo calculado, cuyo resultado es la creación de valor que beneficia a emprendedores, empresas u organizaciones. Este estudio tuvo como objetivo fortalecer la cultura de emprendimiento mediante la investigación como estrategia pedagógica en instituciones educativas. Empleando una metodología basada en el recorrido de las trayectorias de indagación. Las técnicas de recolección de datos fueron el diario de campo y la entrevista semiestructurada. Se realizó un muestreo no probabilístico de tipo intencional para seleccionar la muestra que estuvo conformada por veinticinco (25) estudiantes del grado séptimo con edades comprendidas de once (11) a trece (13) años de la IED John F. Kennedy del municipio de Aracataca, Magdalena. Los resultados arrojaron nuevas perspectivas para desarrollar el emprendimiento con una diversidad de productos elaborados artesanalmente en una variedad de materiales reciclable. Se evidenció la necesidad de fomentar la cultura de emprendimiento en instituciones de educación a través de una disciplina aprovechando los recursos y oportunidades que se pueden generar.

Palabras clave: Cultura de emprendimiento, investigación como estrategia pedagógica, tecnologías de la información y comunicación.

\section{Abstract}

Entrepreneurship culture fosters thoughts and actions, aimed at generating economic income, taking advantage of resources and opportunities, based on the development of a global vision, where a calculated risk must be managed, the result of which is the creation of value that benefits entrepreneurs, companies or organizations. The objective of this study was to strengthen the culture of entrepreneurship through research as a pedagogical strategy in educational institutions. Using a methodology based on the trajectory of the trajectories of inquiry. The data collection techniques were the field diary and the semi-structured interview. A non-probabilistic sampling of intentional type was carried out to select the sample that consisted of twenty-five (25) students of the seventh grade with ages ranging from eleven (11) to thirteen (13) years of the IED John F. Kennedy of the municipality of Aracataca, Magdalena. The results yielded new perspectives to develop the enterprise with a diversity of handcrafted products in a variety of recyclable materials. The need to promote the culture of entrepreneurship in educational institutions was demonstrated through a discipline taking advantage of the resources and opportunities that can be generated.

Keywords: Entrepreneurship culture, research as a pedagogical strategy, information and communication technologies.

1 Líder del grupo de investigación "Formadoras y estrategas de cultura kennedysta".

2 Docentes de la IED John F. Kennedy de Aracataca, sede principal.

- The author; licensee Universidad de la Costa - CUC. 


\section{Introducción}

En Colombia existe un alto índice de pobreza y las instituciones educativas publicas cuentan con una población estudiantil que no supera el estrato 2 , en la IED John F. Kennedy de Aracataca hay estudiantes en condiciones vulnerables, donde muchas veces las familias no cuentan con el dinero suficiente para cubrir las necesidades básicas. El empleo y la educación son derechos fundamentales del ser humano, los cuales buscan garantizar condiciones de vida digna y facilitar el desarrollo económico y social del país, en circunstancias de equidad y de bienestar general (Pereira, 2016), ofreciendo oportunidades que orienten y propicien el desarrollo humano.

Continuando con este orden de ideas, es posible que los estudiantes al terminar la educación básica, estén indecisos frente lo que estudiaran, o quizás no tengan la oportunidad de dirigirse a otra ciudad a iniciar la educación superior, a partir de lo anterior surge la iniciativa de fomentar la cultura de emprendimiento en los estudiantes de la institución, con la finalidad de brindarles herramientas para que ellos mismos puedan aprovechar sus habilidades, capacidades y talentos, para generar una fuente de ingreso económico en sus hogares, mejorando a su vez la calidad de vida del estudiante y sus familias.

El estudio busco generar cultura de emprendimiento en los estudiantes de la institución educativa, donde se utilicen las habilidades artísticas que poseen estos, para la producción de elementos, en diferentes materiales reciclables, que posteriormente puedan ser expuestos en ferias de emprendimiento realizadas por la misma institución, en este punto toma un papel importante el proceso investigativo y las herramientas tecnológicas, que serán utilizadas para indagar so- bre el proceso de producción, materiales o herramientas que necesitan para crear su producto final y lograr por último ser un empresario orientado a emprender nuevas acciones, especialmente en el campo de la economía. (Villalba , Hurtado, Guarín y Casas, 2013)(Saavedra, Urrutia, Paravié, Rohvein y Corres, 2014).

Así mismo se busca que los estudiantes aprendan a utilizar sus finanzas, generar ingresos, invertir y ahorrar mediante la ejecución de proyectos productivos que además según Hernández y Jiménez (2015) debe hacerse de manera ética para que logren permear a las nuevas empresas de estos componentes.

$\mathrm{Al}$ integrar nuevas prácticas pedagógicas, se dinamizan las clases, pero a su vez permiten el fomento de cultura de emprendimiento, mediante el uso de la investigación y las TIC, motivando a los estudiantes a utilizar habilidades y capacidades para mejorar la calidad de vida, donde también se incentiva a la realización de actividades productivas en sus tiempos libres.

Analizando al emprendedor desde diferentes factores globales, se han revisado algunas investigaciones a nivel mundial, a nivel internacional se encontró en Santiago de Chile un proyecto sobre emprendimiento titulado Competencias laborales y prácticas educativas de docentes de enseñanza media técnico profesional". Dicha investigación busco establecer la relación entre las competencias docentes y sus prácticas con las habilidades que desarrollan los educandos de este país. Evaluando los métodos de enseñanza y las perspectivas curriculares para el desarrollo de competencias laborales en los estudiantes (Espinoza, 2007). En otros países como Perú se realizó otra investigación que buscó evaluar la influencia sobre la aplicación y evaluación de un programa de formación para el 
desarrollo de capacidades empresariales y emprendedoras en los estudiantes de grado quinto de educación básica (Córdova y Campoverde, 2008).

A nivel nacional se han realizado estudios como el realizado por la Universidad Javeriana de Bogotá, en estudiantes del primer ciclo de un colegio de la ciudad, con la finalidad de prepararlos para enfrentar con seguridad el desafío y la responsabilidad de ser productivos para sí mismos y para quienes lo rodean, a través de una propuesta pedagógica basada en la investigación (Muñoz y Vera, 2011). Por su parte otro estudio realizado en Bogotá, mostró la experiencia frente la creación de un programa de emprendimiento, donde diseñaron prácticas novedosas como: juegos, simulaciones y creación de comunidades de aprendizaje activo, que permitieron que los estudiantes lograran incorporar el concepto y se motivaran a ser emprendedores, a su vez dicha investigación presento los diferentes programas desarrollados a nivel escolar en diferentes instituciones del país (Flores, s.f).

Otra investigación realizada en el municipio de Cundinamarca se tituló estudio prospectivo sobre los centros de emprendimiento en Colombia: tendencias, escenarios y estrategias en la generación de oportunidades laborales alternativas". Esta presenta información teórica acerca del concepto de emprendimiento visto desde tres disciplinas distintas, como son la Economía, la Sociología y la Psicología; a su vez plantea las características principales del emprendedor y realiza una descripción general de los programas de emprendimiento en Colombia (Peraza, 2010).

El estudio del emprendimiento considerando las condiciones que se desprenden de su desarrollo hacen evidente el creciente interés por el estudio de los procesos que involucran el emprendi- miento y la innovación dentro del plano académico como un campo aplicado a la investigación dentro de los procesos pedagógicos como estrategias de las instituciones educativas y están ligados a los temas de emprendimiento con la disminución de la violencia, desempleo y avance hacia la paz y vinculados a las políticas y estrategias de tipo social en países como Colombia (Cruz y Matiz, 2004).

Colombia ha presentado avances significativos en la calidad de la educación, para esto la secretaria en los últimos años ha venido implementando programas de formación y capacitaciones a docentes y estudiantes de las instituciones educativas, con la finalidad de mejorar la calidad de vida de la comunidad estudiantil. Para contribuir con la mejora en la calidad de la educación se planteó el presente proyecto de investigación, el cual a partir de las sesiones de formación presencial y virtual sobre la IEP apoyada en TIC se consolido y tuvo como objetivo principal, generar cultura de emprendimiento mediante la investigación como estrategia pedagógica en la IED John F. Kennedy de Aracataca, brindando de esta manera una formación integradora con componentes de ciencia, tecnología e innovación, donde a su vez se promovió la cultura de emprendimiento.

Partiendo de los antecedentes de la investigación a nivel nacional e internacional, se observa que hay pocos estudios relacionados con el presente proyecto, que giran en torno al fomento del emprendimiento en instituciones educativas, sin embargo, en este se busca generar la cultura de emprendimiento mediante la investigación como estrategia pedagógica (IEP), donde integrando estos dos componentes a la educación se contribuirá a la generación de nuevas fuentes de empleo formal y de calidad, apoyados en procesos científicos e innovadores. 
Cultura de emprendimiento

mediante la IEP apoyada en TIC

Actualmente en Colombia existen leyes que promueven la inclusión de una formación educativa en cultura de emprendimiento. El Ministerio de Educación Nacional - MEN instauro la ley 1014 del 2006 , la cual invita a los establecimientos educativos a implementar ajustes curriculares, que permitan diseñar propuestas pedagógicas, destinadas a fortalecer actitudes emprendedoras en los estudiantes de educación básica y media. Esta ley surge con la intensión de mejorar el desarrollo social y económico del país, aportando así a mejorar la calidad de vida de los ciudadanos.

En Colombia las instituciones educativas regidas por la normatividad expuesta en la ley 1014 deben incluir en sus planes de estudio la formación en cultura de emprendimiento para mejorar las condiciones de vida de los estudiantes y aportar al desarrollo sostenible del país, por lo cual se busca el desarrollo de la misma con acciones que permitan la formación en competencias básicas, competencias laborales, competencias ciudadanas y competencias empresariales (Ley 1014 de 2006, Art. 1).

Así mismo, la formación sobre emprendimiento proporciona capacidades para crear y hacer crecer una empresa, incrementa las habilidades cognitivas de los individuos para gestionar e identificar las oportunidades y tiene efectos culturales sobre las actitudes y comportamientos de los estudiantes, en la creación de ideas de negocio y el desarrollo de las mismas (Levie y Autio, 2008).

La formación en cultura de emprendimiento implica fomentar competencias emprendedoras las cuales son características individuales como aptitudes y rasgos de personalidad, que puestas en práctica facilitan la adquisición de co- nocimientos, por medio de la educación y de experiencias reales significativas que permitirán resolver problemáticas laborales y sociales que se presentan en la vida en sociedad. Por este motivo, es necesario desarrollar competencias emprendedoras que busquen la autonomía y autorrealización de la persona, como vía para la realización de un proyecto vital (Martínez, 2009).

El propósito del sistema educativo es transformar los medio de una forma expedita y eficaz para transmitir, socializar y recrear la cultura, por lo tanto es desde las instituciones educativas en sus diferentes niveles, que se puede materializar, incluyendo dentro de los sistemas curriculares mediante planes de estudio, con una cultura de emprendimiento dadas mediante un proceso formativo, y desde allí proyectar la generación de oportunidades laborales, a través de las sinergias entre los distintos sectores e instituciones, para consolidar la educación como un derecho y creando las oportunidades para que toda la población se encamine hacia el desarrollo sostenido de la sociedad pertinente a los procesos netamente establecidos (Duarte y Ruiz, 2009).

Partiendo de lo anterior, el espacio más conveniente para la formación en cultura de emprendimiento es la institución educativa, pues esta tiene cobertura en todo el territorio nacional y accede el mayor número de niños, jóvenes y adultos al proceso de formación con una mejor actitud y disposición para el cambio. Por tal razón es primordial la inclusión en los Proyectos Educativos Institucionales PEI el emprendimiento como asignatura, partiendo de los planes de estudio con ventajas como la dotación social de cualidades emprendedoras, avances en la organización social, formación en redes sociales, generación de proyectos de inversión (Duarte \& Ruiz, 2009). 
El emprendimiento es una forma de pensar, razonar y actuar centrada en las oportunidades, planteado con visión global y llevado a cabo mediante un liderazgo equilibrado y la gestión de un riesgo, introducirlo al aula de clases, lo cual permita crear en los estudiantes ideas de negocio que mejoren su estilo de vida y favorezca el desarrollo sostenible de las comunidades (Ley 1014 de 2006, art. $1^{\circ}$ ).

El emprendedor tiene la capacidad de generar ideas y transformar su entorno en algo novedoso, con una actitud que lo diferencia del individuo común. Alrededor del mismo tema Duarte y Ruiz, (2009) plantean cuatro ideas, a través de las cuales articula el hecho con el desarrollo de una cultura de emprendimiento, señalando cuatro factores básicos para crear una empresa:

a. Motivación y determinación: relacionado con el entorno social que rodea al individuo como la familia, la educación, el contexto sociocultural y las políticas de gobierno actuales.

b. Idea y mercado: determina la oportunidad y la viabilidad de negocio en el mercado existente.

c. Recursos: se refiere a la parte económica del proceso, identificación y consecución de recursos tales como: financieros, infraestructura, materiales, tecnológicos y talento humano.

d. Habilidades: relacionado con los conocimientos, habilidades, destrezas y experiencias que debe tener todo empresario. La formación en emprendimiento puede cambiar los atributos que tengan los individuos y puede forjar actitudes emprendedoras en ellos. Promoviendo cualidades psicológicas favorables para la actividad emprendedora, como; autoconfianza, autoestima, autoeficacia y necesidad de alcanzar sus metas (Lapresta, 2013).
Partiendo de lo anterior, el espacio más conveniente para la formación en cultura de emprendimiento es la institución educativa, pues esta tiene cobertura en todo el territorio nacional y accede el mayor número de niños, jóvenes y adultos en proceso de formación con una mejor actitud y disposición para el cambio. Por tal razón es primordial la inclusión en los Proyectos educativos institucionales - PEI del emprendimiento como asignatura, partiendo de los planes de estudio con ventajas como la dotación social de cualidades emprendedoras, avances en la organización social, formación en redes sociales apoyados por las TIC, generación de proyectos de inversión, creación de empresas, generación de autoempleo, por lo tanto se genera desarrollo local, regional y nacional encaminado al mejoramiento de la calidad de vida de las comunidades (Duarte y Ruiz, 2009).

El emprendedor tiene una personalidad con características, rasgos, capacidades que lo impulsan a actuar desde esta perspectiva en mayor o menor grado; haciendo del emprendimiento su forma de pensar, razonar y actuar centrada en las oportunidades, planteado con visión global y llevado a cabo mediante un liderazgo equilibrado y la gestión de un riesgo calculado, su resultado es la creación de valor que beneficia a la empresa, la economía y la sociedad, por lo cual es importante introducirlo al aula de clases, lo cual permita crear en los estudiantes ideas de negocio que mejoren su estilo de vida y favorezca el desarrollo sostenible de las comunidades (Ley 1014, 2006, art. 1).

Ahora bien, el desarrollo sostenible fomenta transformación social en las comunidades, logrando condiciones de vida, cimentando propuestas incluyentes de una forma participativa, mediante el reconocimiento de los derechos de la comunidad pertinente, una perspectiva 
que adquiere sentido, donde la fomente una cultura de emprendimiento encaminados a propuesta que avale espacios de creación e innovación, materializados en oportunidades reales para los seres humanos de ser protagonistas en las propias transformaciones y el mejoramiento de sus contextos (Zambrano, Espitia y Hernández, 2016).

Por su parte, la Investigación como Estrategia Pedagógica, tiene como finalidad instaurar bases científicas a los estudiantes en el aula de clases, esta parte de una dinámica metodológica, que busca dar respuestas a los interrogantes de los niños y niñas de la Institución y que asigna a los docentes un nuevo rol en el aula, para que en el momento indicado, puedan ser ellos mismos quienes busquen comprender el mundo que los rodea e interpretar y describir sus problemas en un lenguaje sustentado en la ciencia (Mariño, 2010),

La educación a lo largo del tiempo ha presentado cambios significativos, que invitan al docente a diseñar e implementar nuevas estrategias pedagógicas para dirigir el proceso de enseñanza con sus estudiantes, una estrategia pedagógica es un conjunto de actividades que se realizan con un orden lógico y coherente en función del cumplimiento de objetivos del plan académico. Es decir, es una planificación que contiene métodos o acciones que permiten al estudiante alcanzar los logros propuestos, mejorar su aprendizaje y facilitar su crecimiento personal (Picardo, Balmore, y Escobar, 2004).

Cochran y Martin (2006) plantearon la existencia de conocimientos y reflexiones en la acción, lo que permite integrar en las actuaciones explícitas e implícitas lo cognitivo, lo emocional, la teoría y la práctica. Reconociendo que tanto quien enseña como quien aprende dentro de una comunidad trabajan para generar conocimiento local, prever su práctica y teorizar sobre ella, interpretando las conclusiones de otros. De ahí que se diseñen estrategias pedagógicas para fortalecer la cultura de emprendimiento en las instituciones educativas ya que ello implica la reconstrucción curricular en esta institución hacia la construcción de un currículo integrado y contextualizado, lo cual pone de manifiesto la discusión pedagógica sobre el problema de la articulación: práctica pedagógica e investigación, específicamente en la función que le corresponde a la investigación en el aula de clase y en la distinción entre dos procesos pedagógicos con identidad propia: investigación y enseñanza.

Una de las finalidades de la educación es capacitar a los estudiantes para comprender, crear y participar en la construcción de su conocimiento, para lo anterior el docente debe cumplir un rol de guía o formador, que a través de estrategias pedagógicas motive al estudiante a alcanzar los logros propuestos, incluir en dichas estrategias las TIC, servirá como una herramienta tecnológica que muestra una forma diferente de organizar, representar y codificar la realidad, además estas son instrumentos que permiten un grado de aplicación de los conocimientos adquiridos. Las tecnologías del aprendizaje representan una de las fuerzas renovadoras en los sistemas de aprendizaje y constituyen un elemento clave para el desarrollo de la educación y la formación (Oleagordia, s.f).

Las TIC conforman el proceso del manejo de la información incluyendo medios y herramientas de manipulación, las TIC se convirtieron en un elemento esencial en los procesos educativos, en la actualidad el internet se implanto como la principal fuente para acceder a la información, donde las herramientas tecnológicas como computadores, tablets y celulares, son una herramienta necesaria para almacenarla y procesarla (Camacho., Fló- 
rez, Gaibao, Aguirre, Pasive y Murcia, G. 2012).

Así mismo, las TIC son tecnologías para almacenar, recuperar, procesar y comunicar la información. Existe una variedad de recursos electrónicos que se encuadran dentro del concepto de TIC como; televisión, teléfonos, videos, ordenadores, entre otros. Sin embargo, los medios más representativos de la sociedad actual son los ordenadores que nos permiten utilizar diferentes aplicaciones informáticas (presentaciones, aplicaciones multimedia, programas ofimáticos y redes de comunicación (Belloch, 2012).

No obstante, el uso de las TIC no implica la implementación de una estrategia de enseñanza/aprendizaje. En algunos casos se producen procesos educativos que integran las TIC siguiendo una metodología tradicional en la que se enfatiza el proceso de enseñanza, donde el estudiante recibe la información del docente. No obstante, para guiar el proceso educativo, se puede fomentar la interacción, el aprendizaje colaborativo y el aprendizaje por descubrimiento utilizando las TIC basados en los diferentes recursos y servicios que ofrece Internet (Belloch, 2012).

De manera que, para realizar el proceso de implementación de las TIC al contexto educativo es necesario tener en cuenta el nivel de madures digital que presentan los usuarios y para esto se debe diseñar un modelo eficaz que permita medir dicho nivel de forma inicial, donde los sujetos observados participen plenamente, asumiendo el ejercicio a partir de la influencia de las TIC en el contexto contemporáneo (Rozo, et al, 2016)

Quienes investigan plantean que la IEP apoyada en las TIC es una propuesta educativa que apunta a mejorar la calidad de la educación, presentando una formación en ciencia, tecnología e innovación. Que permiten el desarrollo de competencias y la creación de nuevo saber y conocimiento el cual va a generar estrategias diversas para afrontar los conflictos escolares, donde a partir de la transversalización de las áreas de estudio las instituciones educativas a través de una metodología didáctica y práctica, favorecen e impactan de manera positiva el proceso enseñanza - aprendizaje, mediante el fortalecimiento de la convivencia escolar.

\section{Metodología}

La metodología del proyecto de investigación se realizó a partir de los recorridos de las trayectorias de indagación, basados en la integración de la IEP apoyada en TIC al aula, articulado al plan de estudios, desde las diferentes áreas se estructuraron temáticas relacionadas con la robótica educativa, la finalidad de este proceso investigativo, fue generar una trasformación social y educativa a través del aprendizaje en contexto, involucrando intereses, motivaciones y realidades de los estudiantes para generar conocimiento científico, donde se establecieron cinco (5) momentos que especifican en la ejecución y las metas trazadas para darle solución a la pregunta problema planteada. A continuación, se presentan los diferentes momentos.

- Momento (1): selección y análisis de la población sujeto de estudio.

- Momento (2): integración de la temática mediante la IEP al aula de clases, se integraron grupos estudiantiles para seleccionar problemáticas relacionadas con la cultura de emprendimiento. Los estudiantes se plantearon preguntas y con el acompañamiento del docente realizaron el proceso de indagación mediante herramientas virtuales para luego realizar el proceso de construcción de sus productos. 
- Momento (3): socialización de los resultados de las indagaciones de estudiantes en una feria institucional dirigida por los docentes a cargo.

- Momento (4): análisis de los resultados de la implementación de la estrategia a través del diario de campo y entrevistas.

- Momento (5): propagación y divulgación de los resultados.

En cuanto a los instrumentos de recolección se utilizó el diario de campo, este es un instrumento que ayuda al investigador a inmiscuirse de forma total en el ambiente con el cual se va a encontrar, es un proceso de hechos, recolección de datos y análisis. El diario de campo es parte de un registro cronológico que se desarrolla mediante la sesión compartida con los infantes, es donde se describe detalladamente los aspectos positivos como negativos colocando significado a cada uno de ellos como un recurso de mejoras en todas las áreas con el grupo a trabajar considerando los recursos manejados y utilizados. Un cuaderno donde se va a descubrir la manera de expresar y escribir frente a la relación que se tiene con los infantes, es un momento de no cohibir lo vivido y las experiencias obtenidas.

Además, se empleó una entrevista estructurada la cual es una técnica de la investigación cualitativa que consiste en realizar preguntas predeterminadas o establecidas con anterioridad sobre el fenómeno a estudiar y sus opciones de respuesta son abiertas, lo cual permite conocer la opinión de la población frente la temática de estudio. El diseño de la entrevista conto con cinco (5) preguntas, tres (3) abiertas y dos (2) cerradas. Las cuales estaban formuladas para conocer los productos que sabían elaborar y los materiales que necesitaban para los mismos, además de indagar sobre la motivación emprendedora y la necesidad de tener una cátedra de emprendimiento en la institución.

La población sujeto de estudio estuvo conformada por 25 estudiantes de la Institución Educativa Departamental John F. Kennedy de Aracataca, con estrato socioeconómico uno (1) y edades que oscilan entre los once (11) y diecisiete (17) años. Se realizó un muestreo no probabilístico de tipo intencional para seleccionar la muestra, seleccionando directa e intencionadamente los individuos de la población. Tomando como criterios de inclusión los estudiantes con habilidades artísticas de la institución.

\section{Resultados}

La búsqueda de esta cultura de emprendimiento en los estudiantes de las instituciones educativas departamentales - IED, llevaron a utilizar las habilidades artísticas que poseen estos, para la producción de elementos artesanales, en diferentes materiales reutilizables; que posteriormente fueron expuestos en ferias de emprendimiento realizadas por la misma institución, en este punto toma un papel importante el proceso investigativo y las herramientas tecnológicas, utilizadas para indagar sobre el proceso de producción, materiales o herramientas que necesitan para crear su producto final.

Así mismo los estudiantes aprendieron a utilizar sus finanzas, generar ingresos, invertir y ahorrar mediante la ejecución de proyectos productivos. Al integrar nuevas prácticas pedagógicas, se dinamizaron las clases, pero a su vez permiten el fomento de cultura de emprendimiento, mediante el uso de la investigación y las TIC, motivando a los estudiantes a utilizar habilidades y capacidades para mejorar la calidad de vida, donde también se incentivaron la realización de actividades productivas en sus tiempos libres. 
A partir de estas actividades culturales de emprendimiento se realizaron entrevistas con la siguiente información resultante de los participantes; encontrando que los estudiantes recurren a las artesanías como fuente primaria de ventas, expresados en productos ubicados en las categorías de decoraciones principalmente; las cuales incluyen flores, mariposas, pavo real, gansos, manzanas, campanas, espejos, pingüinos, portarretratos y libros. Seguidamente elaboraron floreros, pulseras, cofres y productos ubicados en la categoría de lámparas, incluyendo faroles y veleros. Por su parte una minoría realizaron alcancías, sillas y copas; y finalmente unos pocos cestos de ropa, cortinas, escaparates, vitrales, mochilas y colgadores de toallas. Todos inspirados en las siguientes razones que arrojaron las encuestas realizadas:

1. Deseo de generar ingresos de forma autónoma

2. Tomar la decisión de conseguir independencia

3. Aplicar experiencia y conocimientos en un área específica

4. Obtener el capital de trabajo y los recursos físicos para la operación

5. Seleccionar proveedores adecuados en precios, volumen y calidad

6. Asesorarse de expertos en las diversas áreas

7. Innovar continuamente en las áreas seleccionadas

8. Establecer altos estándares de calidad en productos y servicios

9. Crear y mantener una clara visión del futuro.
En cuanto a los materiales empleados para la elaboración de las artesanías presentadas en la feria realizada, se ubica en un $21 \%$ la categoría plásticos (botellas, tapas, cucharas, platos, globos y bolsas). Un 18\% de los estudiantes participantes utilizaron materiales de las categorías papel- cartón y pegante (colbon, cinta, gota mágica y silicona). Y se expresa de manera general con un $61 \%$ de la muestra la implementación de estos diferentes materiales: pintura, tijeras, hilo-nailon, madera, guadua, palo de escoba, pinceles, vidrios, piedras de bisutería, agujas, alambre, icopor y tela.

$\mathrm{Y}$ como punto final de los resultados de la investigación, se recogieron testimonios que demostraron que la población participante del estudio en su totalidad encontraron en la elaboración de productos artesanales una fuente exequible de ingresos sin la necesidad de utilizar recursos de altos costos, los cuales pueden ser impulsados en ferias de emprendimiento en instituciones educativas, además de gustarles la idea de darle importancia a una cátedra de emprendimiento como parte de sus materias escolares.

\section{Conclusiones}

Con la realización de este estudio se puede llegar a la conclusión que las perspectivas de la investigación como estrategia pedagógica en el campo de la cultura de emprendimiento se ha ampliado sustancialmente en esta área, y se puede pretender formular nuevos proyectos de fortalecimiento y desarrollo de esta disciplina donde se den las bases a nuevos esquemas curriculares que permitan la adecuada formación en emprendimiento desde la educación básica en el departamento, teniendo en cuenta que la creación 
y el crecimiento de las organizaciones es un aspecto vital para el progreso de la región y para el horizonte económico de las siguientes generaciones.

Con estas consideraciones se plantea la necesidad de iniciar en las instituciones educativas una cultura de formación emprendedora desde la primaria y habituarla a la sociedad como mecanismo de vida para todas las siguientes generaciones teniendo como objeto potenciar y dinamizar el entorno emprendedor a través de programas que generen habilidades y competencias en los estudiantes que les permitan visualizar el emprendimiento como una alternativa viable, y entrenar, formar y capacitar el desarrollo de las habilidades y competencias para el emprendimiento, generando redes sociales y espacios interdisciplinarios para encuentros de emprendedores intercolegiados.

Lo más pertinente es la implementación, como ya se mencionó antes, en los Proyectos Educativos Institucionales - PEI de las Instituciones Educativas Departamentales - IED, ajustado a los diferentes contextos sin descuidar las orientaciones y desde los Planes Educativos Municipales - PEM y el Plan de Desarrollo Nacional - PDN, así el desarrollo local y regional ampliaría las posibilidades de sus comunidades, capitalizando las oportunidades de las personas para crear e innovar y avanzar en los cambios y el mejoramiento económico y social incrementando la productividad (ONU, 2007).

En ese sentido utilizar la Investigación como Estrategia Pedagógica apoyada en las TIC para fomentar el emprendimiento es una propuesta educativa que apunta a mejorar la calidad de la educación, presentando una formación en ciencia, tecnología e innovación. Además, las TIC posibilitan, afirman Maculan, Jiménez y Castellanos (2015), la adquisición de competencias productivas organizacio- nales, la inserción en diversas redes y la considerable reducción de los riesgos y costos inherentes a su establecimiento en el mercado y a su vez permite el desarrollo de competencias y la creación de nuevo saber y conocimiento el cual va a generar estrategias diversas para afrontar los conflictos escolares, donde a partir de la transversalización de las áreas de estudio las instituciones educativas a través de una metodología didáctica y práctica, favorecen e impactan de manera positiva el proceso enseñanza - aprendizaje, mediante el fortalecimiento de la convivencia escolar.

\section{Referencias}

Belloch. (2012). Las Tecnologías de la Información y Comunicación en el aprendizaje. Valencia: Universidad de Valencia.

Camacho, T., Flórez M., Gaibao, D., Aguirre M., Pasive Y. y Murcia, G. (2012). Estrategias pedagógicas en el ámbito educativo. Bogotá, D.C.

Cochran, M. y Lytle, S. (2003). Más allá de la certidumbre: Adoptar una actitud indagadora sobre la práctica. En, A. Liberman y J. Miller (Eds.). La indagación como base de la formación del profesorado y la mejora de la educación. Barcelona: Octaedro.

Córdova, A. y Campoverde, V. (2008). Influencia del programa de formación "jóvenes en acción" en el desarrollo de las capacidades empresariales en los/ las estudiantes del $5^{\circ}$ grado de educación secundaria del área educación para el trabajo de la Institución Educativa Parcemón Saldarriaga Montejo. ah pachitea. Piura, Perú.

Duarte, T. y Ruiz, M. (2009) Emprendimiento, una opción para el desarrollo. Scientia Et Technica, XV(43). 326331. 
Espinoza, K. (2007). Competencias laborales y prácticas educativas de docentes de enseñanza media técnico profesional. [Tesis de grado]. Santiago, CIUP, Chile.

Flores, A. (s.f). Diseño De Comunidades Emprendedoras, Solidarias Y Democráticas. [Trabajo de campo]. Cátedra de Emprendimiento y Empresariado Solidario para Jóvenes de Municipios de Colombia, Colegio Francisco Julián Olaya, La Mesa (Cundinamarca). Bogotá.

Hernández, H. y Jiménez, A. (2015). Responsabilidad social empresarial, zona urbana del distrito de Barranquilla: estudio etnográfico. Económicas CUC, 36(2), 9-18.

Lapresta, A (2013). Evolucion y desarrollo de los servicios de apoyo a emprendedores y empresas en el ambito del medio rural. Universidad de Valladolid, España.

Levie, J. y Autio E. (2008). A Theoretical grounding and test of the GEM model. Busines Media. LLC

Maculan, A.-M., Jiménez, C. y Castellanos, O. (2015). Aprendizaje en el proceso de incubación de empresas de base tecnológica. Económicas CUC, $36(1), 15-48$.

Mariño, G. (2010). El diálogo en la educación de jóvenes y adultos. Dos propuestas pedagógicas para implementarlo. En, El taller dialógico I la recuperación de experiencias laborales. Bogotá, D.C.: OEI. 2010.

Martínez, F. (2009): Programa SocioEducativo para el desarrollo de la cultura emprendedora entre los jóvenes. Revista Iberoamericana de Educación.

Muñoz, C. y Vera, D. (2011). Fomentando la cultura del emprendimiento en estudiantes del primer ciclo del Colegio
IED Fernando Mazuera Villegas, Sede C. Bogotá, D.C.: Pontificia Universidad Javeriana.

Oleagordia, I. (s.f.). Estrategias educativas para el uso de las nuevas tecnologías de la información y la comunicación. En: Revista Iberoamericana de la educación, $\mathrm{N}^{\circ} 9$.

Pertuz, V., Navarro, A., Rojas, G. y Quintero, L. (2016). Perfil docente y fomento de la cultura del emprendimiento: búsqueda de una relación. Educación y Educadores, 19(1). 29-45.

Pereira, M. (2016). Hacia la construcción de un derecho energético ambiental como disciplina autónoma. Jurídicas CUC, 12(1), 177-204. http://dx.doi. org/10.17981/juridcuc.12.1.2016.10

Picardo, O., Balmore, R. y Escobar, J. (2004). Diccionario enciclopédico de ciencias de la educación. San Salvador: El Salvador.

República de Colombia. Congreso de la República. (26 de enero de 2006). Fomento de la Cultura del Emprendimiento en Colombia. [LEY 1014]. Diario Oficial No. 46.164.

Rozo, J., Martin, A., Fagua, A. y Ávila, Y. (2016). Estrategias de gamificación aplicadas al diagnóstico de la incorporación pedagógica de las TIC en una comunidad académica. Cultura Educación Y Sociedad, 4(1).

Saavedra Sueldo, C., Urrutia, S., Paravié, D., Rohvein, C. y Corres, G. (2014). Una propuesta metodológica para la determinación de capacidades estratégicas en pymes industriales. INGE CUC, 10(2), 43 - 50. Recuperado de https://52.0.212.120/ingecuc/article/ view/489

Villalba, M., Hurtado, H., Guarín, H. y Casas, J. (2013). Innovación en pymes artesanales de Morroa, Sucre, 2012. Económicas CUC, 34(1), 15-28. 
Zambrano, O., Espitia, S. y Hernández, J. (2016). Cultura del emprendimiento en instituciones de educación superior: estrategia de inclusión social. Revista desarrollo gerencial. 